ESAIM: COCV 22 (2016) 338-354

DOI: $10.1051 / \mathrm{cocv} / 2015007$
ESAIM: Control, Optimisation and Calculus of Variations

www.esaim-cocv.org

\title{
EXTERIOR CONVEXITY AND CLASSICAL CALCULUS OF VARIATIONS
}

\author{
Saugata Bandyopadhyay ${ }^{1}$ And Swarnendu Sil ${ }^{2}$
}

\begin{abstract}
We study the relation between various notions of exterior convexity introduced in [S. Bandyopadhyay, B. Dacorogna and S. Sil, J. Eur. Math. Soc. 17 (2015) 1009-1039.] with the classical notions of rank one convexity, quasiconvexity and polyconvexity. To this end, we introduce a projection map, which generalizes the alternating projection for two-tensors in a new way and study the algebraic properties of this map. We conclude with a few simple consequences of this relation which yields new proofs for some of the results discussed in [S. Bandyopadhyay, B. Dacorogna and S. Sil, J. Eur. Math. Soc. 17 (2015) 1009-1039.].
\end{abstract}

Mathematics Subject Classification. 49-XX.

Received July 20, 2014.

Published online March 4, 2016.

\section{INTRODUCTION}

The notion of exterior convexity introduced in [1] is of fundamental importance in calculus of variations on exterior spaces, playing a role similar to what is played by the usual notions of convexity in classical vectorial calculus of variations.

However, the precise connection between these two sets of notions of convexity is a question of somewhat delicate balance. In this article, we explore this connection through the introduction of an appropriate projection map. While this projection map coincides with the canonical alternating projection of the two-tensor fields onto the exterior two-forms, it is non-trivial in the context of higher order forms. Furthermore, the projection map has the crucial property that it projects the tensor product to the exterior product and the gradient to the exterior derivative. It also allows us to express the connection between the notions of exterior convexity and classical notions of convexity in a crisp and explicit way, which is the content of our main theorem stated as follows.

\footnotetext{
Keywords and phrases. Calculus of variations, rank one convexity, quasiconvexity, polyconvexity, exterior convexity, exterior form, differential form.

1 Department of Mathematics \& Statistics, IISER Kolkata, Mohanpur-741246, India. saugata.bandyopadhyay@iiserkol.ac.in

2 Section de Mathématiques, Station 8, EPFL, 1015 Lausanne, Switzerland. swarnendu.sil@epfl.ch
} 
Theorem 1.1. Let $2 \leq k \leq n, f: \Lambda^{k} \rightarrow \mathbb{R}$ and $\pi: \mathbb{R}^{\left(\begin{array}{c}n \\ k-1\end{array}\right) \times n} \rightarrow \Lambda^{k}$ be the projection map. Then the following equivalences hold

$$
\begin{aligned}
f \text { ext. one convex } & \Leftrightarrow f \circ \pi \text { rank one convex. } \\
f \text { ext. quasiconvex } & \Leftrightarrow f \circ \pi \text { quasiconvex. } \\
f \text { ext. polyconvex } & \Leftrightarrow f \circ \pi \text { polyconvex. }
\end{aligned}
$$

The aforementioned result essentially situates the circle of ideas discussed in Bandyopadhyay-DacorognaSil [1] in its proper place with respect to classical calculus of variations, which is well-developed and by now, standard ( $c f$. Dacorogna [3]). It allows us to do calculus of variations back and forth between exterior spaces and the space of matrices. In particular, some results which were directly proved in Bandyopadhya-DacorognaSil [1] turn out be easy corollaries of the theorem aforementioned above, in conjunction with classical results of vectorial calculus of variations. Notable among them is the characterization theorem for ext. quasiaffine functions (compare the proof of Thm. 3.3 in [1] with that of Thm. 3.4). While in this process we do sacrifice the intrinsic character and the co-ordinate free advantage of a direct proof in exterior spaces, a simple proof is obtained nonetheless provided we are ready to assume the results of classical calculus of variations which are non-trivial and technical in their own right.

In this article, our main goal is to prove the aforementioned theorem. While proving the first two equivalences in Theorem 1.1 is easy from the definition of the projection map, proving the third one turns out to be surprizingly difficult and is of our principal concern in this article. One of the obstacles to the proof is the burden of heavy notations. To clarify presentation and to facilitate bookkeeping, we employed a system of notations, which is explained in detail in Section 7 at the end of the article. However, once the cloud of heavy notations is cleared, the proof highlights many intricacies of the algebraic structure of alternating multilinear maps, namely the algebraic structure of determinants and minors and their interrelationship with the algebra of the wedge products which we believe should be of independent interest.

The rest of the paper is organized as follows: in Section 2, we recall the definitions of exterior convexity and introduce the projection map. Section 3 states the main theorem and presents the consequences along with a characterization theorem and a weak lower semicontinuity result. Section 4 explores the algebraic structure of the projection map is greater detail and Section 5 is devoted to the proof of an instrumental lemma, which singles out the crux of the matter. We conclude the proof of the main theorem in Section 6 . Finally, the notations used throughout the article is explained in Section 7.

\section{Preliminaries}

\subsection{Notions of exterior convexity}

We start by recalling the notions of exterior convexity as introduced in [1].

Definition 2.1. Let $1 \leq k \leq n$ and $f: \Lambda^{k} \rightarrow \mathbb{R}$.

(i) We say that $f$ is ext. one convex, if the function

$$
g: t \rightarrow g(t)=f(\xi+t \alpha \wedge \beta)
$$

is convex for every $\xi \in \Lambda^{k}, \alpha \in \Lambda^{k-1}$ and $\beta \in \Lambda^{1}$. If the function $g$ is affine we say that $f$ is ext. one affine. (ii) A Borel measurable and locally bounded function $f$ is said to be ext. quasiconvex, if the inequality

$$
\int_{\Omega} f(\xi+\mathrm{d} \omega) \geq f(\xi) \text { meas } \Omega
$$

holds for every bounded open set $\Omega \subset \mathbb{R}^{n}, \xi \in \Lambda^{k}$ and $\omega \in W_{0}^{1, \infty}\left(\Omega ; \Lambda^{k-1}\right)$. If equality holds, we say that $f$ is ext. quasiaffine. 
(iii) We say that $f$ is ext. polyconvex, if there exists a convex function

$$
F: \Lambda^{k} \times \cdots \times \Lambda^{[n / k] k} \rightarrow \mathbb{R}
$$

such that

$$
f(\xi)=F\left(\xi, \cdots, \xi^{[n / k]}\right), \text { for all } \xi \in \Lambda^{k} .
$$

If $F$ is affine, we say that $f$ is ext. polyaffine.

There are analogous notions of interior convexity ( $c f$. [1]). In what follows, we will discuss the case of exterior convexity only. The case of interior convexity can be derived from the case for exterior convexity by means of Hodge duality.

\subsection{Projection maps}

To study the relationship between the notions introduced in [1] and the classical notions of the vectorial calculus of variations, namely rank one convexity, quasiconvexity and polyconvexity (see [3]), we will introduce a projection map. We first introduce some notations. As usual, by abuse of notations, we identify $\Lambda^{k}\left(\mathbb{R}^{n}\right)$

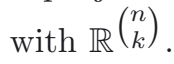

Definition 2.2 (Projection map). Let $2 \leq k \leq n$. We write a matrix $\Xi \in \mathbb{R}^{\left(\begin{array}{c}n \\ k-1\end{array}\right) \times n}$, the upper indices being ordered alphabetically, as

$$
\begin{aligned}
\Xi & =\left(\begin{array}{ccc}
\Xi_{1}^{1 \cdots(k-1)} & \cdots & \Xi_{n}^{1 \cdots(k-1)} \\
\vdots & \ddots & \vdots \\
\Xi_{1}^{(n-k+2) \cdots n} & \cdots & \Xi_{n}^{(n-k+2) \cdots n}
\end{array}\right) \\
& =\left(\Xi_{i}^{I}\right)_{i \in\{1, \cdots, n\}}^{I \in \mathcal{T}^{k-1}}=\left(\begin{array}{c}
\Xi^{1 \cdots(k-1)} \\
\vdots \\
\Xi^{(n-k+2) \cdots n}
\end{array}\right)=\left(\Xi_{1}, \cdots, \Xi_{n}\right) .
\end{aligned}
$$

We define a linear map $\pi: \mathbb{R}^{\left(\begin{array}{c}n \\ k-1\end{array}\right) \times n} \rightarrow \Lambda^{k}\left(\mathbb{R}^{n}\right)$ in the following way

$$
\pi(\Xi)=\sum_{i=1}^{n} \Xi_{i} \wedge \mathrm{e}^{i}
$$

where

$$
\Xi_{i}=\sum_{1 \leq i_{1}<\cdots<i_{k-1} \leq n} \Xi_{i}^{i_{1} \cdots i_{k-1}} \mathrm{e}^{i_{1}} \wedge \cdots \wedge \mathrm{e}^{i_{k-1}}=\sum_{I \in \mathcal{T}^{k-1}} \Xi_{i}^{I} \mathrm{e}^{I}
$$

Remark 2.3. Observe that this projection map can also be written as,

$$
\pi(\Xi)=\sum_{I \in \mathcal{T}^{k}}\left(\sum_{j \in I} \operatorname{sgn}\left(j, I_{j}\right) \Xi_{j}^{I_{j}}\right) \mathrm{e}^{I},
$$

see 3 (vii) in Section 7 for the notations.

\section{Remark 2.4.}

1. Note that the map $\pi: \mathbb{R}^{\left(\begin{array}{c}n \\ k-1\end{array}\right) \times n} \rightarrow \Lambda^{k}\left(\mathbb{R}^{n}\right)$ is onto. 
2. It is easy to see that $\pi: \mathbb{R}^{n \times n} \rightarrow \Lambda^{2}\left(\mathbb{R}^{n}\right)$ is given by

$$
\pi(\xi)=\sum_{i=1}^{n} \xi_{i} \wedge \mathrm{e}^{i}=\sum_{1 \leq i<j \leq n}\left(\xi_{j}^{i}-\xi_{i}^{j}\right) \mathrm{e}^{i} \wedge \mathrm{e}^{j},
$$

so that, with abuse of notation,

$$
\pi(\xi)=\xi-\xi^{T}=2\left(\frac{\xi-\xi^{T}}{2}\right) .
$$

So for $k=2, \pi$ is just twice the alternating projection for 2-tensors (or twice the skew-symmetric projection for square matrices).

\section{Main theorem And CONSEQUences}

\subsection{Main theorem}

The main result of the article is the following:

Theorem 3.1. Let $2 \leq k \leq n, f: \Lambda^{k} \rightarrow \mathbb{R}$ and $\pi: \mathbb{R}^{\left(\begin{array}{c}n \\ k-1\end{array}\right) \times n} \rightarrow \Lambda^{k}$ be the projection map. Then the following equivalences hold

$$
\begin{aligned}
f \text { ext. one convex } & \Leftrightarrow f \circ \pi \text { rank one convex. } \\
f \text { ext. quasiconvex } & \Leftrightarrow f \circ \pi \text { quasiconvex. } \\
f \text { ext. polyconvex } & \Leftrightarrow f \circ \pi \text { polyconvex. }
\end{aligned}
$$

\section{Remark 3.2.}

(i) Note that the theorem does not say that any quasiconvex or rank one convex function $\phi: \mathbb{R}^{\left(\begin{array}{c}n \\ k-1\end{array}\right) \times n} \rightarrow \mathbb{R}$ is of the form $f \circ \pi$ with $f$ ext. quasiconvex or ext. one convex as the following example shows. We let $n=k=2$ and

$$
\phi(\Xi)=\operatorname{det} \Xi
$$

which is clearly polyconvex (and thus quasiconvex and rank one convex). But there is no function $f: \Lambda^{k} \rightarrow \mathbb{R}$ such that $\phi=f \circ \pi$. Indeed if such an $f$ exists, we arrive at a contradiction, since setting

$$
X=\left(\begin{array}{ll}
1 & 0 \\
0 & 1
\end{array}\right) \text { and } Y=\left(\begin{array}{ll}
0 & 0 \\
0 & 0
\end{array}\right),
$$

we have $\pi(X)=\pi(Y)=0$ and thus

$$
1=\phi(X)=f(\pi(X))=f(\pi(Y))=\phi(Y)=0 .
$$

(ii) The following equivalence is, of course, trivially true

$$
f \text { convex } \Leftrightarrow f \circ \pi \text { convex. }
$$

\subsection{Relations between notions of exterior convexity}

We now list a few simple consequences of the main theorem.

Theorem 3.3. Let $1 \leq k \leq n$ and $f: \Lambda^{k} \rightarrow \mathbb{R}$. Then

$$
f \text { convex } \Rightarrow f \text { ext. polyconvex } \Rightarrow f \text { ext. quasiconvex } \Rightarrow f \text { ext. one convex. }
$$


Proof. The result is immediate from Theorem 3.1 and the classical results (cf. [3]). Another, more direct proof, without using the classical results, can be found in [1].

Theorem 3.4. Let $1 \leq k \leq n$ and $f: \Lambda^{k} \rightarrow \mathbb{R}$. The following statements are then equivalent.

(i) $f$ is ext. polyaffine.

(ii) $f$ is ext. quasiaffine.

(iii) $f$ is ext. one affine.

(iv) For every $0 \leq s \leq[n / k]$, there exist $c_{s} \in \Lambda^{k s}$ such that,

$$
f(\xi)=\sum_{s=0}^{[n / k]}\left\langle c_{s} ; \xi^{s}\right\rangle, \text { for every } \xi \in \Lambda^{k} .
$$

Proof. From the definitions of ext. polyaffine functions, it is clear that

$$
\text { (i) } \Leftrightarrow \text { (iv). }
$$

The statements

$$
\text { (i) } \Leftrightarrow \text { (ii) } \Leftrightarrow \text { (iii) }
$$

follow at once from classical results (cf. Thm. 5.20 in [3]) by virtue of Theorem 3.1.

For a more direct proof of the above, see [1]. See also [2] for yet another proof.

Theorem 3.5. Let $1 \leq k \leq n, 1<p<\infty, \Omega \subset \mathbb{R}^{n}$ be a bounded smooth open set and $f: \Lambda^{k} \rightarrow \mathbb{R}$ be ext. quasiconvex verifying, for every $\xi \in \Lambda^{k}$,

$$
c_{1}\left(|\xi|^{p}-1\right) \leq f(\xi) \leq c_{2}\left(|\xi|^{p}+1\right)
$$

for some $c_{1}, c_{2}>0$. if

$$
\alpha_{s} \rightarrow \alpha \quad \text { in } W^{1, p}\left(\Omega ; \Lambda^{k-1}\right)
$$

then

$$
\liminf _{s \rightarrow \infty} \int_{\Omega} f\left(\mathrm{~d} \alpha_{s}\right) \geq \int_{\Omega} f(\mathrm{~d} \alpha)
$$

Proof. According to Theorem 3.1, we have that $f \circ \pi$ is quasiconvex. Then classical results (see Thm. 8.4 in [3]) show that

$$
\liminf _{s \rightarrow \infty} \int_{\Omega} f\left(\mathrm{~d} \alpha_{s}\right)=\liminf _{s \rightarrow \infty} \int_{\Omega} f\left(\pi\left(\nabla \alpha_{s}\right)\right) \geq \int_{\Omega} f(\pi(\nabla \alpha))=\int_{\Omega} f(\mathrm{~d} \alpha)
$$

as wished.

\section{Algebraic properties of the projection}

We now start exploring the algebraic structure of the projection map in greater detail. The following properties are easily obtained. See [4] for a proof.

Proposition 4.1. Let $2 \leq k \leq n$ and $\pi: \mathbb{R}^{\left(\begin{array}{c}n \\ k-1\end{array}\right) \times n} \rightarrow \Lambda^{k}\left(\mathbb{R}^{n}\right)$ be the projection map.

(i) If $\alpha \in \Lambda^{k-1}\left(\mathbb{R}^{n}\right) \sim \mathbb{R}^{\left(\begin{array}{c}n \\ k-1\end{array}\right)}$ and $\beta \in \Lambda^{1}\left(\mathbb{R}^{n}\right) \sim \mathbb{R}^{n}$, then,

$$
\pi(\alpha \otimes \beta)=\alpha \wedge \beta .
$$


(ii) Let $\omega \in C^{1}\left(\Omega ; \Lambda^{k-1}\right)$, then, by abuse of notations,

$$
\pi(\nabla \omega)=\mathrm{d} \omega
$$

The following result is crucial to establish the main theorem in the case of polyconvexity. See Section 5.4 of [3] for the definition of adjugates and Section 7 for the notations.

Proposition 4.2 (Adjugate formula). If $k$ is even, then for $2 \leq s \leq[n / k]$,

$$
[\pi(\Xi)]^{s}=(s !) \sum_{I \in \mathcal{T}^{s k}}\left(\sum_{s}^{I} \operatorname{sgn}(J ; \tilde{I})\left(\operatorname{adj}_{s} \Xi\right)_{J}^{\tilde{I}}\right) \mathrm{e}^{I},
$$

and

$$
[\pi(\Xi)]^{s}=0, \quad \text { for }[n / k]<s \leq \min \left\{n,\left(\begin{array}{c}
n \\
k-1
\end{array}\right)\right\}
$$

If $k$ is odd,

$$
[\pi(\Xi)]^{s}=0, \quad \text { for all } s, 2 \leq s \leq \min \left\{n,\left(\begin{array}{c}
n \\
k-1
\end{array}\right)\right\}
$$

Proof. We prove only the first equality. Everything else follows by properties of the wedge power. So we prove the case when $k$ is even and $2 \leq s \leq[n / k]$. We prove it by induction.

Step 1. To start the induction, we first prove the case when $s=2$.

We have,

$$
\pi(\Xi)=\sum_{I \in \mathcal{T}^{k}}\left(\sum_{j \in I} \operatorname{sgn}\left(j, I_{j}\right) \Xi_{j}^{I_{j}}\right) \mathrm{e}^{I}
$$

So,

$$
\begin{aligned}
& {[\pi(\Xi)]^{2}=\pi(\Xi) \wedge \pi(\Xi)} \\
& =\sum_{I \in \mathcal{T}^{2 k}}\left(\begin{array}{l}
\sum_{\substack{I^{1}, I^{2} \\
I^{1}, I^{2}=I \\
I^{1} \cap I^{2}=\emptyset}} \operatorname{sgn}\left(I^{1}, I^{2}\right)\left(\sum_{j_{1} \in I^{1}} \operatorname{sgn}\left(j_{1}, I_{j_{1}}^{1}\right) \Xi_{j_{1}}^{I_{j_{1}}^{1}}\right) \\
\left.\left(\sum_{j_{2} \in I^{2}} \operatorname{sgn}\left(j_{2}, I_{j_{2}}^{2}\right) \Xi_{j_{2}}^{I_{j_{2}}^{2}}\right)\right)
\end{array}\right) \mathrm{e}^{I}
\end{aligned}
$$


Now, since $k$ is even, we have,

$$
\begin{aligned}
& {[\pi(\Xi)]^{2}} \\
& =2 \sum_{I \in \mathcal{T}^{2 k}}\left(\sum _ { 2 } ^ { I } \left(\operatorname{sgn}\left(\left[j_{1}, I_{j_{1}}^{1}\right],\left[j_{2}, I_{j_{2}}^{2}\right]\right) \operatorname{sgn}\left(j_{1}, I_{j_{1}}^{1}\right) \operatorname{sgn}\left(j_{2}, I_{j_{2}}^{2}\right) \Xi_{j_{1}}^{I_{j_{1}}^{1}} \Xi_{j_{2}}^{I_{j_{2}}^{2}}\right.\right. \\
& \left.\left.+\operatorname{sgn}\left(\left[j_{1}, I_{j_{2}}^{2}\right],\left[j_{2}, I_{j_{1}}^{1}\right]\right) \operatorname{sgn}\left(j_{1}, I_{j_{2}}^{2}\right) \operatorname{sgn}\left(j_{2}, I_{j_{1}}^{1}\right) \Xi_{j_{2}}^{I_{j_{1}}^{1}} \Xi_{j_{1}}^{I_{j_{2}}^{2}}\right)\right) \mathrm{e}^{I} \\
& =2 \sum_{I \in \mathcal{T}^{2 k}}\left(\sum_{2}^{I}\left(\operatorname{sgn}\left(j_{1}, I_{j_{1}}^{1}, j_{2}, I_{j_{2}}^{2}\right) \Xi_{j_{1}}^{I_{j_{1}}^{1}} \Xi_{j_{2}}^{I_{j_{2}}^{2}}+\operatorname{sgn}\left(j_{1}, I_{j_{2}}^{2}, j_{2}, I_{j_{1}}^{1}\right) \Xi_{j_{2}}^{I_{j_{1}}^{1}} \Xi_{j_{1}}^{I_{j_{2}}^{2}}\right)\right) \mathrm{e}^{I} \\
& =2 \sum_{I \in \mathcal{T}^{2 k}}\left(\sum_{2}^{I} \operatorname{sgn}\left(j_{1}, I_{j_{1}}^{1}, j_{2}, I_{j_{2}}^{2}\right)\left(\Xi_{j_{1}}^{I_{j_{1}}^{1}} \Xi_{j_{2}}^{I_{j_{2}}^{2}}-\Xi_{j_{2}}^{I_{j_{1}}^{1}} \Xi_{j_{1}}^{I_{j_{2}}^{2}}\right)\right) \mathrm{e}^{I} \\
& =2 \sum_{I \in \mathcal{T}^{2 k}}\left(\sum_{2}^{I} \operatorname{sgn}\left(j_{1}, I_{j_{1}}^{1}, j_{2}, I_{j_{2}}^{2}\right)\left(\operatorname{adj}_{2} \Xi\right)_{j_{1} j_{2}}^{I_{j_{1}}^{1} I_{j_{2}}^{2}}\right) \mathrm{e}^{I},
\end{aligned}
$$

which proves the case for $s=2$.

Step 2. We assume the result to be true for some $s \geq 2$ and show that it holds for $s+1$, thus completing the induction. Now we know, by Laplace expansion formula for the determinants,

$$
\begin{aligned}
\left(\operatorname{adj}_{s+1} \Xi\right)_{j_{1} \ldots j_{s+1}}^{I^{1} \ldots I^{s+1}} & =\sum_{m=1}^{s+1} \Xi_{j_{l}}^{I^{m}}(-1)^{l+m}\left(\operatorname{adj}_{s} \Xi\right)_{j_{1} \ldots \hat{j}_{l} \ldots j_{s+1}}^{I^{1} \ldots \widehat{I^{m}} \ldots I^{s+1}}, \text { for any } 1 \leq l \leq s+1 \\
& =\frac{1}{s+1} \sum_{l=1}^{s+1} \sum_{m=1}^{s+1} \Xi_{j_{l}}^{I^{m}}(-1)^{l+m}\left(\operatorname{adj}_{s} \Xi\right)_{j_{1} \ldots \hat{j}_{l} \ldots j_{s+1}}^{I^{1} \ldots \widehat{I^{m}} \ldots I^{s+1}} .
\end{aligned}
$$

Note that, for any $1 \leq l, m \leq s+1$,

$$
\operatorname{sgn}\left(j_{1}, I^{1}, \ldots, j_{s+1}, I^{s+1}\right)=(-1)^{\{(l-1)+(m-1)(k-1)\}} \operatorname{sgn}\left(j_{l}, I^{m}, \tilde{I}^{l, m}\right),
$$

where $\tilde{I}^{l, m}$ is a shorthand for the permutation $\left(\tilde{j}_{1}, \tilde{I}^{1}, \ldots, \tilde{j}_{s}, \tilde{I}^{s}\right)$ and

- $\tilde{j}_{1}<\ldots<\tilde{j}_{s}$ and $\left\{\tilde{j}_{1}, \ldots, \tilde{j}_{s}\right\}=\left\{j_{1}, \ldots, \widehat{j_{l}}, \ldots, j_{s+1}\right\}$.

- $\tilde{I}^{1}<\ldots<\tilde{I}^{s}$ and $\left\{\tilde{I}^{1}, \ldots, \tilde{I}^{s}\right\}=\left\{I^{1}, \ldots, \widehat{I^{m}}, \ldots, I^{s+1}\right\}$.

Note that this means $\tilde{j}_{r}=j_{r}$ for $1 \leq r<l$ and $\tilde{j}_{r}=j_{r+1}$ for $l \leq r \leq s$. Similarly, $\tilde{I}^{r}=I^{r}$ for $1 \leq r<m$ and $\tilde{I}^{r}=I^{r+1}$ for $m \leq r \leq s$. Now since $k$ is even, for any $1 \leq l, m \leq s+1$,

$$
\begin{aligned}
& \operatorname{sgn}\left(j_{1}, I^{1}, \ldots, j_{s+1}, I^{s+1}\right)=(-1)^{l+m} \operatorname{sgn}\left(j_{l}, I^{m}, \tilde{I}^{l, m}\right) \\
& =(-1)^{l+m} \operatorname{sgn}\left(j_{l}, I^{m}\right) \operatorname{sgn}\left(\tilde{I}^{l m}\right) \operatorname{sgn}\left(\left[j_{l}, I^{m}\right],\left[\tilde{I}^{l, m}\right]\right) .
\end{aligned}
$$

Thus,

$$
\begin{aligned}
& \operatorname{sgn}\left(j_{1}, I^{1}, \ldots, j_{s+1}, I^{s+1}\right)\left(\operatorname{adj}_{s+1} \Xi\right)_{j_{1} \ldots j_{s+1}}^{I^{1} \ldots I^{s+1}} \\
& =\frac{1}{(s+1)} \sum_{l, m=1}^{s+1} \operatorname{sgn}\left(\left[j_{l}, I^{m}\right],\left[\tilde{I}^{l, m}\right]\right) \operatorname{sgn}\left(j_{l}, I^{m}\right) \Xi_{j_{l}}^{I^{m}} \operatorname{sgn}\left(\tilde{I}^{l m}\right)\left(\operatorname{adj}_{s} \Xi\right)_{j_{1} \ldots \hat{j}_{l} \ldots j_{s+1}}^{I^{1} \ldots \widehat{I}^{m}} .
\end{aligned}
$$


Hence,

$$
\begin{aligned}
& (s+1) ! \sum_{I \in \mathcal{T}^{(s+1) k}}\left(\sum_{s+1}^{I} \operatorname{sgn}\left(j_{1}, I^{1}, \ldots, j_{s+1}, I^{s+1}\right)\left(\operatorname{adj}_{s+1} \Xi\right)_{j_{1} \ldots j_{s+1}}^{I^{1} \ldots I^{s+1}}\right) \mathrm{e}^{I} \\
& =\frac{(s+1) !}{(s+1)} \sum_{I \in \mathcal{T}^{(s+1) k}}\left(\begin{array}{r}
\sum_{s+1}^{I} \sum_{l, m=1}^{s+1} \operatorname{sgn}\left(\left[j_{l}, I^{m}\right],\left[\tilde{I}^{l, m}\right]\right) \operatorname{sgn}\left(j_{l}, I^{m}\right) \Xi_{j_{l}}^{I^{m}} \\
\operatorname{sgn}\left(\tilde{I}^{l m}\right)\left(\operatorname{adj}_{s} \Xi\right)_{j_{1} \ldots \hat{j}_{l} \ldots j_{s+1}}^{I^{1} \ldots \widehat{I}^{m}} \ldots I^{s+1} \\
\end{array}\right) \mathrm{e}^{I}
\end{aligned}
$$

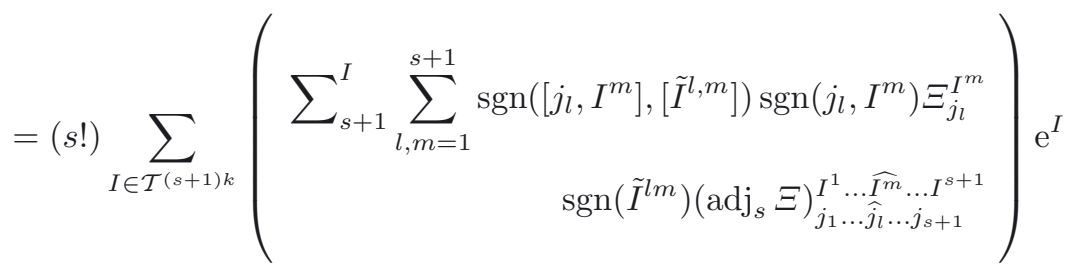

$$
\begin{aligned}
& =\sum_{I \in \mathcal{T}^{(s+1) k}}\left(\begin{array}{c}
\sum_{\substack{I^{\prime} \subset I \\
I^{\prime} \in \mathcal{T}^{k}}}\left(\operatorname{sgn}\left(I^{\prime},\left[I \backslash I^{\prime}\right]\right)\left(\sum_{j \in I^{\prime}} \operatorname{sgn}\left(j, I_{j}^{\prime}\right) \Xi_{j}^{I_{j}^{\prime}}\right)\right. \\
\left.\left(s !\left(\sum_{s}^{\left[I \backslash I^{\prime}\right]} \operatorname{sgn}\left(\tilde{j}_{1}, \tilde{I}^{1}, \ldots, \tilde{j}_{s}, \tilde{I}^{s}\right)\left(\operatorname{adj}_{s} \Xi\right)_{\tilde{j}_{1} \ldots \tilde{j}_{s}^{1}} \ldots \tilde{I}^{s}\right)\right)\right)
\end{array}\right) \mathrm{e}^{I},
\end{aligned}
$$

where the last line is just a rewriting of the penultimate one. Indeed on expanding the sums the map, sending $j_{l}$ to $j ; I^{m}$ to $I_{j}^{\prime} ; I^{1}, \ldots, \widehat{I^{m}}, \ldots, I^{s+1}$ to $\tilde{I}^{1}, \ldots, \tilde{I}^{s}$ respectively and $j_{1}, \ldots, \hat{j}_{l}, \ldots, j_{s+1}$ to $\tilde{j}_{1}, \ldots, \tilde{j}_{s}$ respectively is a bijection between the terms on the two sides of the last equality.

So, we have, by induction hypothesis,

$$
\begin{aligned}
& (s+1) ! \sum_{I \in \mathcal{T}^{(s+1) k}}\left(\sum_{s+1}^{I} \operatorname{sgn}\left(j_{1}, I^{1}, \ldots, j_{s+1}, I^{s+1}\right)\left(\operatorname{adj}_{s+1} \Xi\right)_{j_{1} \ldots j_{s+1}}^{I^{1} \ldots I^{s+1}}\right) \mathrm{e}^{I} \\
& =\sum_{I \in \mathcal{T}^{(s+1) k}}\left(\begin{array}{c}
\sum_{\substack{I^{\prime} \subset I \\
I^{\prime} \in \mathcal{T}^{k}}} \operatorname{sgn}\left(I^{\prime},\left[I \backslash I^{\prime}\right]\right)\left(\text { coefficient of } \mathrm{e}^{I^{\prime}} \text { in } \pi(\Xi)\right) \\
\times\left(\text { coefficient of } \mathrm{e}^{\left[I \backslash I^{\prime}\right]} \text { in }[\pi(\Xi)]^{s}\right)
\end{array}\right) \mathrm{e}^{I} \\
& =\sum_{I \in \mathcal{T}^{(s+1) k}}\left(\text { coefficient of } \mathrm{e}^{I} \text { in }[\pi(\Xi)]^{s+1}\right) \mathrm{e}^{I}=[\pi(\Xi)]^{s+1} \text {. }
\end{aligned}
$$

This completes the induction proving the desired result.

Since we have seen that $[\pi(\Xi)]^{s}$ depends only on $\operatorname{adj}_{s} \Xi$, we are now in a position to define a linear projection for every value of $s$. These maps will be useful later.

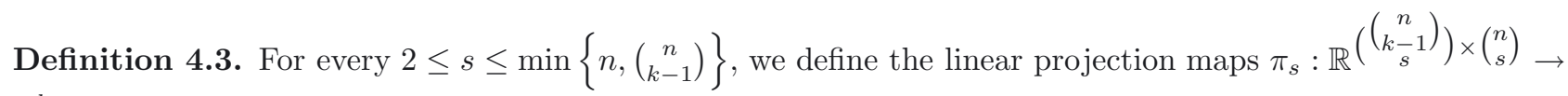
$\Lambda^{k s}\left(\mathbb{R}^{n}\right)$ by the condition,

$$
\pi_{s}\left(\operatorname{adj}_{s}(\Xi)\right)=[\pi(\Xi)]^{s} \text { for all } \Xi \in \mathbb{R}^{\left(\begin{array}{c}
n \\
k-1
\end{array}\right) \times n} .
$$


Remark 4.4. It is clear that this condition uniquely defines the projection maps. For the sake of consistency, we define, $\pi_{1}=\pi$ and $\pi_{0}$ is defined to be the identity map from $\mathbb{R}$ to $\mathbb{R}$.

\section{An important Lemma}

Lemma 5.1. Let $2 \leq k \leq n$ and $N=\left(\begin{array}{c}n \\ k-1\end{array}\right)$. Consider the function

$$
g(X, d)=f(\pi(X))-\sum_{s=1}^{\min \{N, n\}}\left\langle d_{s}, \operatorname{adj}_{s} X\right\rangle
$$

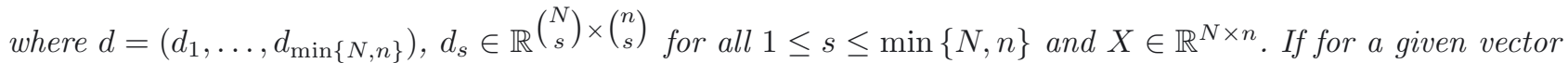
$d$, the function $X \mapsto g(X, d)$ achieves a minimum over $\mathbb{R}^{N \times n}$, then for all $1 \leq s \leq \min \{N, n\}$, there exists $\mathcal{D}_{s} \in \Lambda^{k s}$ such that,

$$
\left\langle d_{s}, \operatorname{adj}_{s} Y\right\rangle=\left\langle\mathcal{D}_{s}, \pi_{s}\left(\operatorname{adj}_{s} Y\right)\right\rangle \quad \text { for all } Y \in \mathbb{R}^{N \times n} .
$$

The lemma is technical and quite heavy in terms of notations. So before proceeding to prove the lemma as stated, it might be helpful to spell out the idea of the proof. The plan is always the same. In short, if the conclusion of the lemma does not hold, we can always choose a matrix $X$ such that $g(X, d)$ can be made to be smaller than any given real number, contradicting the hypothesis that the map $X \mapsto g(X, d)$ assumes a minimum.

Proof. Let us fix a vector $d$ and assume that for this $d$, the function $X \mapsto g(X, d)$ achieves a minimum over $\mathbb{R}^{N \times n}$.

We will first show that all adjugates with a common index between subscripts and superscripts must have zero coefficients. More precisely, we claim that,

Claim 5.2. For any $2 \leq k \leq n$ and for every $1 \leq s \leq \min \{N, n\}$, for every $J \in \mathcal{T}^{s}, I=\left\{I^{1} \ldots I^{s}\right\}$ where $I^{1}, \ldots, I^{s} \in \mathcal{T}^{k-1}$, we have,

$$
\left(d_{s}\right)_{J}^{I}=0 \text { whenever } I \cap J \neq \emptyset .
$$

We prove Claim 5.2, using induction over $s$. To start the induction, we first show the case $s=1$. Let $j \in I$, where $I \in \mathcal{T}^{k-1}$. We choose $X=\lambda \mathrm{e}^{j} \otimes \mathrm{e}^{I}$, then clearly $\pi(X)=0$. Also, $g(X, d)=f(0)-\lambda\left(d_{1}\right)_{j}^{I}$. By letting $\lambda$ to $+\infty$ and $-\infty$ respectively, we deduce that $\left(d_{1}\right)_{j}^{I}=0$, since otherwise we obtain a contradiction to the fact that $g$ achieves a finite minima.

Now we assume that Claim 5.2 holds for all $1 \leq s \leq p$ and prove the result for $s=p+1$. We consider $\left(d_{p+1}\right)_{j_{1} \ldots j_{p+1}}^{I^{1} \ldots I^{p+1}}$ with $j_{l} \in I^{m}$ for some $1 \leq l, m \leq p+1$.

Now we first order the rest of the indices (other than the common index) in subscripts and the rest of the multiindices (other than the one with the common index) in superscripts. Let $\tilde{I}^{1}<\ldots<\tilde{I}^{p}$ and $\tilde{j}_{1}<\ldots<\tilde{j}_{p}$ represent the multiindices and indices in the sets $\left\{I^{1}, \ldots, I^{p+1}\right\} \backslash\left\{I^{m}\right\}$ and $\left\{j_{1}, \ldots, j_{p+1}\right\} \backslash\left\{j_{l}\right\}$ respectively.

Now we choose,

$$
X=\lambda \mathrm{e}^{j_{l}} \otimes \mathrm{e}^{I^{m}}+\sum_{r=1}^{p} \mathrm{e}^{\tilde{j}_{r}} \otimes \mathrm{e}^{\tilde{I}^{r}} .
$$

Since $j_{l} \in I^{m}$, we get $\pi(X)$ is independent of $\lambda$. Also, all lower order non-constant adjugates of $X$ must contain the index $j_{l}$ both in subscript and in superscript and hence their coefficients are 0 by the induction hypothesis. Hence, the only non-constant adjugate of $X$ appearing in the expression for $g(X, d)$ is,

$$
\left(\operatorname{adj}_{p+1} X\right)_{j_{1} \ldots j_{p+1}}^{I^{1} \ldots I^{p+1}}=(-1)^{\alpha} \lambda
$$


where $\alpha$ is a fixed integer. Now,

$$
g(X, d)=(-1)^{\alpha+1} \lambda\left(d_{p+1}\right)_{j_{1} \ldots j_{p+1}}^{I^{1} \ldots I^{p+1}}+\text { constants. }
$$

Again as before, we let $\lambda$ to $+\infty$ and $-\infty$ and we deduce, by the same argument, $\left(d_{p+1}\right)_{j_{1} \ldots j_{p+1}}^{I^{1} \ldots I^{p+1}}=0$. This completes the induction and proves the claim.

At this point we split the proof in two cases, the case when $k$ is an even integer and the case when $k$ is an odd integer.

\section{Case 1: $\mathrm{k}$ is even}

Note that, unless $k=2$, it does not follow from above that $d_{s}=0$ for all $s \geq\left[\frac{n}{k}\right]$. The possibility that two different blocks of multiindices in the superscript have some index in common has not been ruled out. Now we will show that the coefficients of two different adjugates having the same set of indices are related in the following way:

Claim 5.3. For every $s \geq 1$,

$$
\operatorname{sgn}(J ; I)\left(d_{s}\right)_{J}^{I}=\operatorname{sgn}(\tilde{J} ; \tilde{I})\left(d_{s}\right)_{\tilde{J}}^{\tilde{I}},
$$

whenever $J \cup I=\tilde{J} \cup \tilde{I}$, with $J, \tilde{J} \in \mathcal{T}^{s}, I=\left\{I^{1} \ldots I^{s}\right\}=\left[I^{1}, \ldots, I^{s}\right], \tilde{I}=\left\{\tilde{I}^{1} \ldots \tilde{I}^{s}\right\}=\left[\tilde{I}^{1}, \ldots, \tilde{I}^{s}\right]$, $I^{1}, \ldots, I^{s}, \tilde{I}^{1}, \ldots, \tilde{I}^{s} \in \mathcal{T}^{k-1}$ and $J \cap I=\emptyset$. In particular, given any $U \in \mathcal{T}^{k s}$, there exists a constant $\mathcal{D}_{U} \in \mathbb{R}$ such that,

$$
\operatorname{sgn}(J ; I)\left(d_{s}\right)_{J}^{I}=\mathcal{D}_{U},
$$

for all $J \cup I=U$ with $J \in \mathcal{T}^{s}, I=\left\{I^{1} \ldots I^{s}\right\}=\left[I^{1}, \ldots, I^{s}\right], I^{1}, \ldots, I^{s} \in \mathcal{T}^{k-1}$.

We will prove the claim again by induction over $s$. We first prove it for the case $s=1$.

For the case $s=1$, we just need to prove, for any index $j$, any multindex $I \in \mathcal{T}^{k-1}$ such that $j \cap I=\emptyset$, we have

$$
\operatorname{sgn}(j, I)\left(d_{1}\right)_{j}^{I}=\operatorname{sgn}(\tilde{j}, \tilde{I})\left(d_{1}\right)_{\tilde{j}}^{\tilde{I}},
$$

where $[j, I]=[\tilde{j}, \tilde{I}]$. We choose $X=\lambda \operatorname{sgn}(j, I) \mathrm{e}^{j} \otimes \mathrm{e}^{I}-\lambda \operatorname{sgn}(\tilde{j}, \tilde{I}) \mathrm{e}^{\tilde{j}} \otimes \mathrm{e}^{\tilde{I}}$. Clearly, $\pi(X)=0$ and this gives,

$$
g(X, d)=f(0)+\lambda\left(\operatorname{sgn}(j, I)\left(d_{1}\right)_{j}^{I}-\operatorname{sgn}(\tilde{j}, \tilde{I})\left(d_{1}\right)_{\tilde{j}}^{\tilde{I}}\right),
$$

where we have used Claim 5.2 to deduce that $\left(d_{2}\right)_{[j \tilde{j}]}^{[I \tilde{I}]}=0$. Letting $\lambda$ to $+\infty$ and $-\infty$, we get $(5.2)$.

Now we assume the result for all $1 \leq s \leq s_{0}$ and show it for $s=s_{0}+1$. Let $\left[I^{1} \ldots I^{s_{0}+1} j_{1} \ldots j_{s_{0}+1}\right]=$ $\left[\tilde{I}^{1} \ldots \tilde{I}^{s_{0}+1} \tilde{j}_{1} \ldots \tilde{j}_{s_{0}+1}\right]$. Note that the sets $\left\{I^{1} \ldots I^{s_{0}+1} j_{1} \ldots j_{s_{0}+1}\right\}$ and $\left\{\tilde{I}^{1} \ldots \tilde{I}^{s_{0}+1} \tilde{j}_{1} \ldots \tilde{j}_{s_{0}+1}\right\}$ are permutations of each other, preserving an order relation given by $j_{1}<\ldots<j_{s_{0}+1}, \tilde{j}_{1}<\ldots<\tilde{j}_{s_{0}+1}, I^{1}<\ldots<I^{s_{0}+1}$ and $\tilde{I}^{1}<\ldots<\tilde{I}^{s_{0}+1}$. Thus the aforementioned sets can be related by any permutation (of $k\left(s_{0}+1\right)$ indices) that respects this order. Since any such permutation is a product of $k$-flips, it is enough to prove the claim in case of $k$-flips ( $c f$. Def. 7.2).

We now assume $(J, I)$ and $(\tilde{J}, \tilde{I})$ are related by a $k$-flip interchanging the subscript $j_{l}$ with one index in the superscript block $I^{m}$ and keep all the other indices unchanged. Also, we assume that after the interchange, the position of the multiindex containing $j_{l}$ in the superscript is $p$ and the new position of the index from the multiindex $I^{m}$ in the subscript is q, i.e., $j_{l} \in \tilde{I}^{p}$ and $\tilde{j}_{q} \in I^{m}$. We also order the remaining indices and assume,

$$
\breve{I}=\left[\breve{I}^{1}, \ldots, \breve{I}^{s_{0}}\right]=\left\{\breve{I}^{1} \ldots \breve{I}^{s_{0}}\right\}=\left\{I^{1} \ldots \widehat{I^{m}} \ldots I^{s_{0}+1}\right\}
$$

and

$$
\breve{J}=\left[\breve{j}_{1} \ldots \breve{j}_{s_{0}}\right]=\left\{\breve{j}_{1} \ldots \breve{j}_{s_{0}}\right\}=\left\{j_{1} \ldots \widehat{j}_{l} \ldots j_{s_{0}+1}\right\}
$$


respectively. Now we choose,

$$
X=\lambda \operatorname{sgn}\left(j_{l}, I^{m}\right) \mathrm{e}^{j_{l}} \otimes \mathrm{e}^{I^{m}}-\lambda \operatorname{sgn}\left(\tilde{j}_{q}, \tilde{I}^{p}\right) \mathrm{e}^{\tilde{j}_{q}} \otimes \mathrm{e}^{\tilde{I}^{p}}+\sum_{1 \leq r \leq s_{0}} \mathrm{e}^{\breve{j}_{r}} \otimes \mathrm{e}^{\breve{I}_{r}} .
$$

Note that $\pi(X)$ is independent of $\lambda$. Also, all non-constant adjugates of $X$ appearing with possibly nonzero coefficients in the expression for $g(X, d)$ have, either $j_{l}$ in subscript and $I^{m}$ in superscript or has $\tilde{j}_{q}$ as a subscript and $\tilde{I}^{p}$ as a superscript, but never both as then they have zero coefficients by Claim 5.2. Also, these adjugates occur in pairs. More precisely, for every non-constant adjugate of $X$ appearing with possibly non-zero coefficients in the expression for $g(X, d)$ having $j_{l}$ in subscript and $I^{m}$ in superscript, there is one having $\tilde{j}_{q}$ in subscript and $\tilde{I}^{p}$ in superscript.

Let us show that, for any $1 \leq s \leq s_{0}+1$, any subset $\bar{J}_{s-1}=\left\{\bar{j}_{1}, \ldots, \bar{j}_{s-1}\right\} \subset \breve{J}$ of $s$ indices and any choice of of $s-1$ multiindices $\bar{I}^{1}, \ldots, \bar{I}^{s-1}$ out of $s_{0}$ multiindices $\breve{I}^{1}, \ldots, \breve{I}^{s_{0}}$, we have,

$$
\frac{\left(\operatorname{adj}_{s} X\right)_{\left[j_{l}, \bar{I}_{s-1}\right]}^{\left[I^{m}, \ldots \bar{I}^{s-1}\right]}}{\operatorname{sgn}\left(\left[j_{l} \bar{J}_{s-1}\right] ;\left[I^{m}, \bar{I}^{1}, \ldots, \bar{I}^{s-1}\right]\right)}=-\frac{\left(\operatorname{adj}_{s} X\right)_{\left[\tilde{j}_{q} \bar{J}_{s-1}\right]}^{\left[\tilde{I}^{p} \bar{I}^{1}, \ldots, \bar{I}^{s-1}\right]}}{\operatorname{sgn}\left(\left[\tilde{j}_{q} \bar{J}_{s-1}\right] ;\left[\tilde{I}^{p}, \bar{I}^{1}, \ldots, \bar{I}^{s-1}\right]\right)} .
$$

Let $a_{1}$ be the position of $j_{l}$ in $\left[j_{l} \bar{J}_{s-1}\right], a_{2}$ be the position of $\tilde{j}_{q}$ in $\left[\tilde{j}_{q} \bar{J}_{s-1}\right], b_{1}$ be the position of $I^{m}$ in $\left[I^{m}, \bar{I}^{1}, \ldots, \bar{I}^{s-1}\right]$ and $b_{2}$ be the position of $\tilde{I}^{p}$ in $\left[\tilde{I}^{p}, \bar{I}^{1}, \ldots, \bar{I}^{s-1}\right]$.

Since $k$ is even,

$$
\begin{aligned}
& \operatorname{sgn}\left(\left[j_{l} \bar{J}_{s-1}\right] ;\left[I^{m}, \bar{I}^{1}, \ldots, \bar{I}^{s-1}\right]\right) \\
& =(-1)^{\left\{\left(a_{1}-1\right)+\left(b_{1}-1\right)\right\}} \operatorname{sgn}\left(j_{l}, I^{m}\right) \operatorname{sgn}\left(\bar{J}_{s-1} ;\left\{\bar{I}^{1} \ldots \bar{I}^{s-1}\right\}\right) \\
& \times \operatorname{sgn}\left(\left[j_{l}, I^{m}\right],\left[\left(\bar{J}_{s-1} ;\left\{\bar{I}^{1} \ldots \bar{I}^{s-1}\right\}\right)\right]\right),
\end{aligned}
$$

and

$$
\begin{aligned}
& \operatorname{sgn}\left(\left[\tilde{j}_{q} \bar{J}_{s-1}\right] ;\left[\tilde{I}^{p}, \bar{I}^{1}, \ldots, \bar{I}^{s-1}\right]\right) \\
& =(-1)^{\left\{\left(a_{2}-1\right)+\left(b_{2}-1\right)\right\}} \operatorname{sgn}\left(\tilde{j}_{q}, \tilde{I}^{p}\right) \operatorname{sgn}\left(\bar{J}_{s-1} ;\left\{\bar{I}^{1} \ldots \bar{I}^{s-1}\right\}\right) \\
& \times \operatorname{sgn}\left(\left[\tilde{j}_{q}, \tilde{I}^{p}\right],\left[\left(\bar{J}_{s-1} ;\left\{\bar{I}^{1} \ldots \bar{I}^{s-1}\right\}\right)\right]\right) .
\end{aligned}
$$

We also have,

$$
\left(\operatorname{adj}_{s} X\right)_{\left[j_{l} \bar{J}_{s-1}\right]}^{\left[I^{m}, \bar{I}^{1}, \ldots, \bar{I}^{s-1}\right]}=(-1)^{a_{1}+b_{1}} \operatorname{sgn}\left(j_{l}, I^{m}\right) \lambda\left(\operatorname{adj}_{s-1} X\right)_{\left[\bar{J}_{s-1}\right]}^{\left[\bar{I}^{1}, \ldots, \bar{I}_{s-1}\right]},
$$

and

$$
\left(\operatorname{adj}_{s} X\right)_{\left[\tilde{j}_{q}, \bar{I}_{s-1}, \ldots, \bar{I}^{s-1}\right]}^{\left[\tilde{I}^{p}\right]}=-(-1)^{a_{2}+b_{2}} \operatorname{sgn}\left(\tilde{j}_{q}, \tilde{I}^{p}\right) \lambda\left(\operatorname{adj}_{s-1} X\right)_{\left[\bar{J}_{s-1}, \ldots, \bar{I}_{s-1}\right]}^{\left[\bar{I}^{1}\right.} .
$$

Combining the four equations above, the result follows.

We now finish the proof of Claim 5.3. Using (5.3), we have,

$$
\begin{array}{r}
g(X, d)=\lambda\left\{\begin{array}{c}
(-1)^{\alpha}\left(\operatorname{sgn}(J ; I)\left(d_{s_{0}+1}\right)_{J}^{I}-\operatorname{sgn}(\tilde{J} ; \tilde{I})\left(d_{s_{0}+1}\right)_{\tilde{J}}^{\tilde{I}}\right) \\
+\sum_{s=1}^{s_{0}} \sum^{s} k_{s, \gamma}\left(\begin{array}{c}
\operatorname{sgn}\left(\left[j_{l} \bar{J}_{s-1}\right] ;\left[I^{m}, \bar{I}^{1}, \ldots, \bar{I}^{s-1}\right]\right)\left(d_{s}\right)_{\left[j_{l}, \bar{I}^{1}, \ldots, \bar{I}^{s-1}\right]}^{\left[I_{s-1}^{m}\right]} \\
-\operatorname{sgn}\left(\left[\tilde{j}_{q} \bar{J}_{s-1}\right] ;\left[\tilde{I}^{p}, \bar{I}^{1}, \ldots, \bar{I}^{s-1}\right]\right)\left(d_{s}\right)_{\left[\tilde{j}_{q}, \bar{I}^{1}, \ldots, \bar{I}^{s-1}\right]}^{\left[\bar{J}_{s-1}\right]} \\
+ \text { constants }
\end{array}\right)
\end{array}\right\}
\end{array}
$$


where $\sum^{s}$ is a shorthand, for every $1 \leq s \leq s_{0}$, for the sum over all possible such choices of $\bar{J}_{s-1}, \bar{I}^{1}, \bar{I}^{2}, \ldots, \bar{I}^{s-1}$ and $k_{s, \gamma}$ is a generic placeholder for the constants appearing before each term of the sum and $\alpha$ is an integer.

By the induction hypothesis, the sum on the right hand side of the above expression is 0 . Hence, we obtain,

$$
g(X, d)=(-1)^{\alpha} \lambda\left(\operatorname{sgn}(J ; I)\left(d_{s_{0}+1}\right)_{J}^{I}-\operatorname{sgn}(\tilde{J} ; \tilde{I})\left(d_{s_{0}+1}\right)_{\tilde{J}}^{\tilde{I}}\right)+\text { constants. }
$$

Letting $\lambda$ to $+\infty$ and $-\infty$, the claim is proved by induction.

Note that by virtue of Claims 5.3 and 5.2 now implies, that for every $1 \leq s \leq \min \{N, n\}$, for every $J \in \mathcal{T}^{s}, I=\left\{I^{1} \ldots I^{s}\right\}$ where $I^{1}, \ldots, I^{s} \in \mathcal{T}^{k-1}$, we have,

$$
\left(d_{s}\right)_{J}^{I}=0 \text { whenever either } I \cap J \neq \emptyset \text { or } I^{l} \cap I^{m} \neq \emptyset \text { for some } 1 \leq l<m \leq s .
$$

Indeed, if $I \cap J \neq \emptyset$, we are done, using Claim 5.2. So let us assume $I \cap J=\emptyset$ but $I^{l} \cap I^{m} \neq \emptyset$ for some $1 \leq l<m \leq s$. Then there exists an index $i$ such that $i \in I^{l}$ and $i \in I^{m}$, we consider the $k$-flip interchanging some index $j$ from subscript with the index $i$ in $I^{l}$. More precisely, let $\tilde{J} \in \mathcal{T}^{s}$ and $\tilde{I}^{l} \in \mathcal{T}^{k-1}$ be such that $i \in \tilde{J}$, $\tilde{J} \backslash\{i\} \subset J, I^{l} \backslash\{i\} \subset \tilde{I}^{l}$ and $J \cup I^{l}=\tilde{J} \cup \tilde{I}^{l}$, then by Claim 5.3 we have,

$$
\operatorname{sgn}(J ; I)\left(d_{s}\right)_{J}^{I}=\operatorname{sgn}\left(\tilde{J} ;\left[\tilde{I}^{l}, I^{1}, \ldots, \widehat{I}^{l}, \ldots, I^{s}\right]\right)\left(d_{s}\right)_{\tilde{J}}^{\left[\tilde{I}^{l}, I^{1}, \ldots, \widehat{I}^{l}, \ldots, I^{s}\right]} .
$$

Since, $i \in \tilde{J}$ and $i \in I^{m}, \tilde{J} \cap\left[\tilde{I}^{l}, I^{1}, \ldots, \widehat{I^{l}}, \ldots, I^{s}\right] \neq \emptyset$, the right hand side of above equation is 0 and so $\left(d_{s}\right)_{J}^{I}=0$, which proves (5.4). So this now implies, $d_{s}=0$ for all $s \geq\left[\frac{n}{k}\right]$. Hence we have, using (5.1), (5.4) and Proposition 4.2,

$$
\begin{aligned}
\left\langle d_{s}, \operatorname{adj}_{s} Y\right\rangle & =\sum_{I \in \mathcal{T}^{s k}} \sum_{s}^{I}\left(d_{s}\right)_{J}^{\tilde{I}}\left(\operatorname{adj}_{s} Y\right)_{J}^{\tilde{I}} \\
& =\sum_{I \in \mathcal{T}^{s k}} \sum_{s}^{I} \operatorname{sgn}(J ; \tilde{I})\left(d_{s}\right)_{J}^{\tilde{I}} \operatorname{sgn}(J ; \tilde{I})\left(\operatorname{adj}_{s} Y\right)_{J}^{\tilde{I}} \\
& =\sum_{I \in \mathcal{T}^{s k}} \frac{1}{s !} \mathcal{D}_{I} \sum_{s}^{I}(s !) \operatorname{sgn}(J ; \tilde{I})\left(\operatorname{adj}_{s} Y\right)_{J}^{\tilde{I}} \\
& =\left\langle\mathcal{D}_{s}, \pi_{s}\left(\operatorname{adj}_{s} Y\right)\right\rangle,
\end{aligned}
$$

where $\mathcal{D}_{s}=\frac{1}{s !} \sum_{I \in \mathcal{T}^{s k}} \mathcal{D}_{I} \mathrm{e}^{I}$, which finishes the proof when $k$ is even.

\section{Case 3: $\mathrm{k}$ is odd}

In this case, by Proposition 4.2, it is enough to show that all coefficients of all terms, except the linear ones must be zero. As in the case above, the plan is to establish a relation between the coefficients of two different adjugates having the same set of indices. But when $k$ is odd, the relationship is not as nice as in the even case and as such there is no general formula. However, we still have a weaker analogue of claim 5.3 for the case of $k$-flips.

Claim 5.4. For $s \geq 1$, if $J, \tilde{J} \in \mathcal{T}^{s}$, and $I^{1} \ldots, I^{s}, \tilde{I}^{1}, \ldots, \tilde{I}^{s} \in \mathcal{T}^{k-1}$, where $J=\left\{j_{1} \ldots j_{s}\right\}, \tilde{J}=\left\{\tilde{j}_{1} \ldots \tilde{j}_{s}\right\}$, $I=\left\{I^{1} \ldots I^{s}\right\}=\left[I^{1}, \ldots, I^{s}\right]$ and $\tilde{I}=\left\{\tilde{I}^{1} \ldots \tilde{I}^{s}\right\}=\left[\tilde{I}^{1}, \ldots, \tilde{I}^{s}\right]$ be such that $J \cap I=\emptyset$ and $(J, I)$ and $(\tilde{J}, \tilde{I})$ are related by a $k$-flip interchanging an index $j_{l}$ in the subscript with one from the multiindex $I^{m}$ in the superscript. Also, we assume that after the interchange, the position of the multiindex containing $j_{l}$ in the superscript is $p$ and the new position of the index from the multiindex $I^{m}$ in the subscript is $q$, i.e., $j_{l} \in \tilde{I}^{p}$ and $\tilde{j}_{q} \in I^{m}$.

Then we have,

$$
\operatorname{sgn}(J ; I)\left(d_{s}\right)_{J}^{I}=(-1)^{(m-p)} \operatorname{sgn}(\tilde{J} ; \tilde{I})\left(d_{s}\right)_{\tilde{J}}^{\tilde{I}}
$$


Since the proof of Claim 5.4 is very similar to that of Claim 5.3, we shall indicate only a brief sketch of the proof. Since $k$ is odd, we deduce,

$$
\begin{gathered}
\operatorname{sgn}\left(\left[j_{l} \bar{J}_{s-1}\right] ;\left[I^{m}, \bar{I}^{1}, \ldots, \bar{I}^{s-1}\right]\right)=(-1)^{\left\{\left(a_{1}-1\right)\right\}} \operatorname{sgn}\left(j_{l}, I^{m}\right) \operatorname{sgn}\left(\bar{J}_{s-1} ;\left\{\bar{I}^{1} \ldots \bar{I}^{s-1}\right\}\right) \\
\times \operatorname{sgn}\left(\left[j_{l}, I^{m}\right],\left[\left(\bar{J}_{s-1} ;\left\{\bar{I}^{1} \ldots \bar{I}^{s-1}\right\}\right)\right]\right), \\
\operatorname{sgn}\left(\left[\tilde{j}_{q} \bar{J}_{s-1}\right] ;\left[\tilde{I}^{p}, \bar{I}^{1}, \ldots, \bar{I}^{s-1}\right]\right)=(-1)^{\left\{\left(a_{2}-1\right)\right\}} \operatorname{sgn}\left(\tilde{j}_{q}, \tilde{I}^{p}\right) \operatorname{sgn}\left(\bar{J}_{s-1} ;\left\{\bar{I}^{1} \ldots \bar{I}^{s-1}\right\}\right) \\
\times \operatorname{sgn}\left(\left[\tilde{j}_{q}, \tilde{I}^{p}\right],\left[\left(\bar{J}_{s-1} ;\left\{\bar{I}^{1} \ldots \bar{I}^{s-1}\right\}\right)\right]\right),
\end{gathered}
$$

and hence, in a manner analogous to the proof of (5.3), we have,

$$
\frac{\left(\operatorname{adj}_{s} X\right)_{\left[j_{l}\right.}^{\left[j^{m}, \bar{I}_{s-1}^{1}, \ldots, \bar{I}^{s-1}\right]}}{\operatorname{sgn}\left(\left[j_{l} \bar{J}_{s-1}\right] ;\left[I^{m}, \bar{I}^{1}, \ldots, \bar{I}^{s-1}\right]\right)}=-(-1)^{\left(b_{1}-b_{2}\right)} \frac{\left(\operatorname{adj}_{s} X\right)_{\left[\tilde{j}_{q}\right.}^{\left[\tilde{I}^{p}, \bar{J}^{1}, \ldots, \bar{I}^{s-1}\right]}}{\operatorname{sgn}\left(\left[\tilde{j}_{q} \bar{J}_{s-1}\right] ;\left[\tilde{I}^{p}, \bar{I}^{1}, \ldots, \bar{I}^{s-1}\right]\right)},
$$

for any $1 \leq s \leq s_{0}+1$, any subset $\bar{J}_{s-1}=\left\{\bar{j}_{1}, \ldots, \bar{j}_{s-1}\right\} \subset \breve{J}$ of $s-1$ indices and any choice of of $s$ multiindices $\bar{I}^{1}, \ldots, \bar{I}^{s-1}$ out of $s_{0}+1$ multiindices, where $a_{1}$ is the position of $j_{l}$ in $\left[j_{l} \bar{J}_{s-1}\right], a_{2}$ is the position of $\tilde{j}_{q}$ in $\left[\tilde{j}_{q} \bar{J}_{s-1}\right], b_{1}$ is the position of $I^{m}$ in $\left[I^{m}, \bar{I}^{1}, \ldots, \bar{I}^{s-1}\right]$ and $b_{2}$ is the position of $\tilde{I}^{p}$ in $\left[\tilde{I}^{p}, \bar{I}^{1}, \ldots, \bar{I}^{s-1}\right]$. Claim 5.4 follows from above.

Note that Claims 5.4 and 5.2 together now rule out the possibility that an adjugate with non-zero coefficient can have common indices between the blocks of multiindices in the superscript and proves $d_{s}=0$ for all $s>\left[\frac{n}{k}\right]$. Furthermore, by Claim 5.4, the coefficients of any two adjugates $\left(d_{s}\right)_{J}^{I},\left(d_{s}\right)_{\tilde{J}}^{\tilde{I}}$ such that $I \cup J=\tilde{I} \cup \tilde{J}$, can differ only by a sign. So clearly, all of them must be 0 if one of them is. So without loss of generality, we shall restrict our attention to the coefficient of a particularly ordered adjugates, one with all distinct indices in subscript and superscripts, for which $j_{1}<\ldots<j_{s}<i_{1}^{1}<\ldots<i_{k-1}^{1}<\ldots<i_{1}^{s}<\ldots<i_{k-1}^{s}$, henceforth referred to as the totally ordered adjugate, Hence for a given $s, 2 \leq s \leq\left[\frac{n}{k}\right]$, and given $\mathcal{I} \in \mathcal{T}^{k s}$, we shall show that,

$$
\left(d_{s}\right)_{j_{1} j_{2} \ldots j_{s}}^{\left\{i_{1}^{1} i_{2}^{1} \ldots i_{k-1}^{1}\right\}\left\{i_{1}^{2} i_{2}^{2} \ldots i_{k-1}^{2}\right\} \ldots\left\{i_{1}^{s} i_{2}^{s} \ldots i_{k-1}^{s}\right\}}=0
$$

where $j_{1}<\ldots<j_{s}<i_{1}^{1}<\ldots<i_{k-1}^{1}<\ldots<i_{1}^{s}<\ldots<i_{k-1}^{s}$. To prove (5.6), we first need the following:

Claim 5.5. For any $1 \leq r \leq k-1$, we have,

$$
\begin{aligned}
& \left(d_{s}\right)_{j_{1} j_{2} \ldots j_{s}}^{\left\{i_{1}^{1} i_{2}^{1} \ldots i_{r}^{1} i_{r+1}^{2} i_{r+2}^{2} \ldots i_{k-1}^{2}\right\}\left\{i_{r+1}^{1} i_{r+2}^{1} \ldots i_{k-1}^{1} i_{1}^{2} i_{2}^{2} \ldots i_{r}^{2}\right\} \ldots\left\{i_{1}^{s} i_{2}^{s} \ldots i_{k-1}^{s}\right\}} \\
& =-\left(d_{s}\right)_{j_{1} j_{2} \ldots j_{s}}^{\left\{i_{1}^{1} i_{2}^{1} \ldots i_{k-1}^{1}\right\}\left\{i_{1}^{2} i_{2}^{2} \ldots i_{k-1}^{2}\right\} \ldots\left\{i_{1}^{s} i_{2}^{s} \ldots i_{k-1}^{s}\right\}} .
\end{aligned}
$$

We prove the claim by induction over $r$. The case for $r=1$ follows from repeated applications of Claim 5.4 as follows.

Using Claim 5.4 to the $k$-flip interchanging $j_{1}$ and $i_{1}^{1}$, then to the $k$-flip interchanging $i_{1}^{1}$ and $i_{1}^{2}$ and finally to the $k$-flip interchanging $j_{1}$ and $i_{1}^{2}$, we get,

$$
\begin{aligned}
& \left(d_{s}\right)_{j_{1} j_{2} \ldots j_{s}}^{\left\{i_{1}^{1} i_{2}^{1} \ldots i_{k-1}^{1}\right\}\left\{i_{1}^{2} i_{2}^{2} \ldots i_{k-1}^{2}\right\} \ldots\left\{i_{1}^{s} i_{2}^{s} \ldots i_{k-1}^{s}\right\}} \\
& =(-1)^{s}\left(d_{s}\right)_{j_{2} \ldots j_{s} i_{1}^{1}}^{\left\{j_{1} i_{2}^{1} \ldots i_{k-1}^{1}\right\}\left\{i_{1}^{2} i_{2}^{2} \ldots i_{k-1}^{2}\right\} \ldots\left\{i_{1}^{s} i_{2}^{s} \ldots i_{k-1}^{s}\right\}} \\
& =-(-1)^{s}\left(d_{s}\right)_{j_{2} \ldots j_{s} i_{1}^{2}}^{\left\{j_{1} i_{k-1}^{1} \ldots i_{1}^{1}\right\}\left\{i_{1}^{1} i_{2}^{2} \ldots i_{k-1}^{2}\right\} \ldots\left\{i_{1}^{s} i_{2}^{s} \ldots i_{k-1}^{s}\right\}} \\
& \left.=-(-1)^{s}(-1)^{s-2}\left(d_{s}\right)_{j_{1} j_{2} \ldots j_{s}}^{\left\{i_{1}^{1} i_{i}^{2} \ldots i_{k-1}^{2}\right.}\right\}\left\{i_{2}^{1} i_{2}^{1} \ldots i_{k-1}^{1} i_{1}^{2}\right\} \ldots\left\{i_{1}^{s} i_{2}^{s} \ldots i_{k-1}^{s}\right\} \text {. }
\end{aligned}
$$

This proves the case for $r=1$. 
We now assume that (5.7) is true for $1 \leq r \leq r_{0}-1$ and show the result for $r=r_{0}$. To show this, it is enough to prove that for any $2 \leq r_{0} \leq k-1$,

$$
\begin{aligned}
& \left(d_{s}\right)_{j_{1} j_{2} \ldots j_{s}}^{\left\{i_{1}^{1} i_{2}^{1} \ldots i_{r_{0}-1}^{1} i_{r_{0}}^{2} i_{r_{0}+1}^{2} \ldots i_{k-1}^{2}\right\}\left\{i_{r_{0}}^{1} i_{r_{0}+1}^{1} \ldots i_{k-1}^{1} i_{1}^{2} i_{2}^{2} \ldots i_{r_{0}-1}^{2}\right\} \ldots\left\{i_{1}^{s} i_{2}^{s} \ldots i_{k-1}^{s}\right\}} \\
& =\left(d_{s}\right)_{j_{1} j_{2} \ldots j_{s}}^{\left.i_{1}^{1} i_{2}^{1} \ldots i_{r_{0}-1}^{1} i_{r_{0}}^{1} i_{r_{0}+1}^{2} \ldots i_{k-1}^{2}\right\}\left\{i_{r_{0}+1}^{1} i_{r_{0}+2}^{1} \ldots i_{k-1}^{1} i_{1}^{2} i_{2}^{2} \ldots i_{r_{0}}^{2}\right\} \ldots\left\{i_{1}^{s} i_{2}^{s} \ldots i_{k-1}^{s}\right\}} .
\end{aligned}
$$

Indeed the result for $r=r_{0}$ follows by combining the induction hypothesis and (5.8). The proof is similar to the case for $r=1$. Indeed, by applying Claim 5.4 to the $k$-flip interchanging $j_{1}$ and $i_{r_{0}}^{1}$, then to the $k$-flip interchanging $i_{r_{0}}^{1}$ and $i_{r_{0}}^{2}$ and finally to the $k$-flip interchanging $j_{1}$ and $i_{r_{0}}^{2}$, we deduce,

$$
\begin{aligned}
& \left(d_{s}\right)_{j_{1} j_{2} \ldots j_{s}}\left\{i_{1}^{1} \ldots i_{r_{0}-1}^{1} i_{r_{0}}^{2} \ldots i_{k-1}^{2}\right\}\left\{i_{r_{0}}^{1} i_{r_{0}+1}^{1} \ldots i_{k-1}^{1} i_{1}^{2} \ldots i_{r_{0}-1}^{2}\right\} \ldots\left\{i_{1}^{s} i_{2}^{s} \ldots i_{k-1}^{s}\right\} \\
& =(-1)^{s-1}\left(d_{s}\right)_{j_{2} \ldots j_{s} i_{r_{0}}^{1}}^{\left\{j_{1} i_{r_{0}+1}^{1} \ldots i_{k-1}^{1} i_{1}^{2} \ldots i_{r_{0}-1}^{2}\right\}\left\{i_{1}^{1} \ldots i_{r_{0}-1}^{1} i_{r_{0}}^{2} \ldots i_{k-1}^{2}\right\} \ldots\left\{i_{1}^{s} i_{2}^{s} \ldots i_{k-1}^{s}\right\}} \\
& =-(-1)^{s-1}\left(d_{s}\right)_{j_{2} \ldots j_{s} i_{r_{0}}^{2}}^{\left\{j_{1} i_{r_{1}+1}^{1} \ldots i_{k-1}^{1} i_{1}^{2} \ldots i_{r_{0}-1}^{2}\right\}\left\{i_{1}^{1} \ldots i_{r_{0}}^{1} i_{r_{0}+1}^{2} \ldots i_{k-1}^{2}\right\} \ldots\left\{i_{1}^{s} i_{2}^{s} \ldots i_{k-1}^{s}\right\}} \\
& =-(-1)^{s-1}(-1)^{s-2}\left(d_{s}\right)_{j_{1} j_{2} \ldots j_{s}}^{\left\{i_{1}^{1} \ldots i_{r_{0}}^{1} i_{r_{0}+1}^{2} \ldots i_{k-1}^{2}\right\}\left\{i_{r_{0}+1}^{1} \ldots i_{k-1}^{1} i_{1}^{2} \ldots i_{r_{0}}^{2}\right\} \ldots\left\{i_{1}^{s} i_{2}^{s} \ldots i_{k-1}^{s}\right\}} .
\end{aligned}
$$

This proves (5.8)) and establishes Claim 5.5.

Now, using Claim 5.5, in particular for $r=k-1$, we obtain,

$$
\left(d_{s}\right)_{j_{1} j_{2} \ldots j_{s}}^{\left\{i_{1}^{1} i_{i}^{1} \ldots i_{k-1}^{1}\right\}\left\{i_{1}^{2} i_{2}^{2} \ldots i_{k-1}^{2}\right\} \ldots\left\{i_{1}^{s} i_{2}^{s} \ldots i_{k-1}^{s}\right\}}=-\left(d_{s}\right)_{j_{1} j_{2} \ldots j_{s}}^{\left\{i_{1}^{1} i_{2}^{1} \ldots i_{k-1}^{1}\right\}\left\{i_{1}^{2} i_{2}^{2} \ldots i_{k-1}^{2}\right\} \ldots\left\{i_{1}^{s} i_{2}^{s} \ldots i_{k-1}^{s}\right\}} .
$$

This proves (5.6) and finishes the proof of the lemma in the case when $k$ is odd and thereby establishes Lemma 5.1 in all cases.

\section{Proof OF THE MAIN THEOREM}

We start by recalling a result regarding ext. polyconvex functions which we will use later. See [1] (cf. Prop. 14(ii)) for the proof.

Proposition 6.1. Let $f: \Lambda^{k} \rightarrow \mathbb{R}$. Then $f$ is ext. polyconvex if and only if, for every $\xi \in \Lambda^{k}$ and $1 \leq s \leq[n / k]$, there exists $c_{s}=c_{s}(\xi) \in \Lambda^{k s}$ such that

$$
f(\eta) \geq f(\xi)+\sum_{s=1}^{[n / k]}\left\langle c_{s}(\xi) ; \eta^{s}-\xi^{s}\right\rangle, \quad \text { for every } \eta \in \Lambda^{k}
$$

Now we are ready to prove the main theorem.

\section{Proof of Theorem 3.1.}

(i) Recall (cf. Prop. 4.1) that

$$
\pi(\alpha \otimes \beta)=\alpha \wedge \beta
$$

The rank one convexity of $f \circ \pi$ follows then at once from the ext. one convexity of $f$. We now prove the converse. Let $\xi \in \Lambda^{k}, \alpha \in \Lambda^{k-1}$ and $\beta \in \Lambda^{1}$; we have to show that

$$
g: t \rightarrow g(t)=f(\xi+t \alpha \wedge \beta)
$$

is convex. Since the map $\pi$ is onto, we can find $\Xi \in \mathbb{R}^{\left(\begin{array}{c}n \\ k-1\end{array}\right) \times n}$ so that $\pi(\Xi)=\xi$. Therefore,

$$
g(t)=f(\pi(\Xi)+t \pi(\alpha \otimes \beta))=f(\pi(\Xi+t \alpha \otimes \beta)),
$$

and the convexity of $g$ follows at once from the rank one convexity of $f \circ \pi$. 
(ii) Similarly since ( $c f$. Prop. 4.1) $\pi(\nabla \omega)=\mathrm{d} \omega$, we immediately infer the quasiconvexity of $f \circ \pi$ from the ext. quasiconvexity of $f$. The reverse implication follows also in the same manner.

(iii) Since $f$ is ext. polyconvex we can find, using Proposition 6.1 , for every $\alpha \in \Lambda^{k}$ and $1 \leq s \leq\left[\frac{n}{k}\right], c_{s}=$ $c_{s}(\alpha) \in \Lambda^{k s}$, such that

$$
f(\beta) \geq f(\alpha)+\sum_{s=1}^{[n / k]}\left\langle c_{s}(\alpha) ; \beta^{s}-\alpha^{s}\right\rangle, \quad \text { for every } \beta \in \Lambda^{k} .
$$

Appealing to Proposition 4.2 we get, for every $\xi \in \mathbb{R}^{\left(\begin{array}{c}n \\ k-1\end{array}\right) \times n}$,

$$
\begin{aligned}
f(\pi(\eta)) & \geq f(\pi(\xi))+\sum_{s=1}^{[n / k]}\left\langle c_{s}(\pi(\xi)) ;[\pi(\eta)]^{s}-[\pi(\xi)]^{s}\right\rangle \\
& =f(\pi(\xi))+\sum_{s=1}^{[n / k]}\left\langle\widetilde{c}_{s}(\xi) ; \operatorname{adj}_{s} \eta-\operatorname{adj}_{s} \xi\right\rangle,
\end{aligned}
$$

for every $\eta \in \mathbb{R}^{\left(\begin{array}{c}n \\ k-1\end{array}\right) \times n}$, which shows that $f \circ \pi$ is indeed polyconvex by Theorem 5.6 in [3].

We now prove the reverse implication. Take $N=\left(\begin{array}{c}n \\ k-1\end{array}\right)$. Since $f \circ \pi$ is polyconvex, we have, using Theorem 5.6

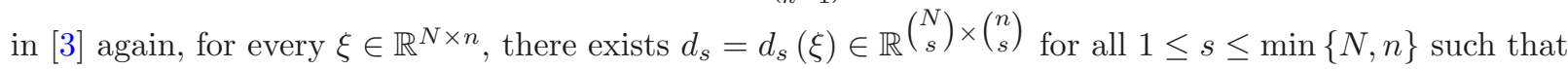

$$
f(\pi(\eta)) \geq f(\pi(\xi))+\sum_{s=1}^{\min \{N, n\}}\left\langle d_{s}(\xi) ; \operatorname{adj}_{s} \eta-\operatorname{adj}_{s} \xi\right\rangle
$$

for every $\eta \in \mathbb{R}^{N \times n}$.

But this means that there exists $d$, given by $d=\left(d_{1}, \ldots, d_{\min \{N, n\}}\right)$ such that the function $X \mapsto g(X, d)$, where $g(X, d)$ is as defined in Lemma 5.1 , achieves a minima at $X=\xi$. Then Lemma 5.1 implies, for every $1 \leq s \leq \min \{N, n\}$, there exists $\mathcal{D}_{s} \in \Lambda^{k s}$ such that

$$
\left\langle d_{s}, \operatorname{adj}_{s} \eta-\operatorname{adj}_{s} \xi\right\rangle=\left\langle\mathcal{D}_{s} ; \pi_{s}\left(\operatorname{adj}_{s} \eta\right)-\pi_{s}\left(\operatorname{adj}_{s} \xi\right)\right\rangle,
$$

for every $\eta \in \mathbb{R}^{N \times n}$. Hence, we obtain from (6.1), for every $\xi \in \mathbb{R}^{N \times n}$,

$$
f(\pi(\eta)) \geq \quad f(\pi(\xi))+\sum_{s=1}^{[n / k]}\left\langle\mathcal{D}_{s}(\xi) ; \pi_{s}\left(\operatorname{adj}_{s} \eta\right)-\pi_{s}\left(\operatorname{adj}_{s} \xi\right)\right\rangle
$$

for every $\eta \in \mathbb{R}^{N \times n}$. Since $\pi$ is onto, given any $\alpha, \beta \in \Lambda^{k}$, we can find $\eta, \xi \in \mathbb{R}^{N \times n}$ such that $\pi(\eta)=\beta$ and $\pi(\xi)=\alpha$. Now using (6.2) and the definition of $\pi_{s}$, we have, by defining $c_{s}(\alpha)=\mathcal{D}_{s}(\xi)$, for every $\alpha \in \Lambda^{k}$,

$$
f(\beta) \geq f(\alpha)+\sum_{s=1}^{[n / k]}\left\langle c_{s}(\alpha) ; \beta^{s}-\alpha^{s}\right\rangle, \quad \text { for every } \beta \in \Lambda^{k} .
$$

This proves $f$ is ext. polyconvex and concludes the proof of the theorem.

\section{Notations}

We gather here the notations which we have used throughout this article. 
1) Let $k$ be a nonnegative integer and $n$ be a positive integer.

- We write $\Lambda^{k}\left(\mathbb{R}^{n}\right)$ (or simply $\Lambda^{k}$ ) to denote the vector space of all alternating $k$-linear maps $f: \underbrace{\mathbb{R}^{n} \times \cdots \times \mathbb{R}^{n}}_{k \text {-times }} \rightarrow$ $\mathbb{R}$. For $k=0$, we set $\Lambda^{0}\left(\mathbb{R}^{n}\right)=\mathbb{R}$. Note that $\Lambda^{k}\left(\mathbb{R}^{n}\right)=\{0\}$ for $k>n$ and, for $k \leq n, \operatorname{dim}\left(\Lambda^{k}\left(\mathbb{R}^{n}\right)\right)=\left(\begin{array}{l}n \\ k\end{array}\right)$.

- $\wedge,\lrcorner,\langle;\rangle$ and $*$ denote the exterior product, the interior product, the scalar product and the Hodge star operator respectively.

- If $\left\{\mathrm{e}^{1}, \cdots, \mathrm{e}^{n}\right\}$ is a basis of $\mathbb{R}^{n}$, then, identifying $\Lambda^{1}$ with $\mathbb{R}^{n}$,

$$
\left\{\mathrm{e}^{i_{1}} \wedge \cdots \wedge \mathrm{e}^{i_{k}}: 1 \leq i_{1}<\cdots<i_{k} \leq n\right\}
$$

is a basis of $\Lambda^{k}$. An element $\xi \in \Lambda^{k}\left(\mathbb{R}^{n}\right)$ will therefore be written as

$$
\xi=\sum_{1 \leq i_{1}<\cdots<i_{k} \leq n} \xi_{i_{1} i_{2} \cdots i_{k}} \mathrm{e}^{i_{1}} \wedge \cdots \wedge \mathrm{e}^{i_{k}}=\sum_{I \in \mathcal{T}^{k}} \xi_{I} \mathrm{e}^{I}
$$

where

$$
\mathcal{T}^{k}=\left\{I=\left(i_{1}, \cdots, i_{k}\right) \in \mathbb{N}^{k}: 1 \leq i_{1}<\cdots<i_{k} \leq n\right\} .
$$

An element of $\mathcal{T}^{k}$ will be referred to as a multiindex. We adopt the alphabetical order for comparing two multiindices and we do not reserve a specific symbol for this ordering. The usual ordering symbols, when written in the context of multiindices will denote alphabetical ordering.

- We write

$$
\mathrm{e}^{i_{1}} \wedge \cdots \wedge \widehat{\mathrm{e}^{i_{s}}} \wedge \cdots \wedge \mathrm{e}^{i_{k}}=\mathrm{e}^{i_{1}} \wedge \cdots \wedge \mathrm{e}^{i_{s-1}} \wedge \mathrm{e}^{i_{s+1}} \wedge \cdots \wedge \mathrm{e}^{i_{k}} .
$$

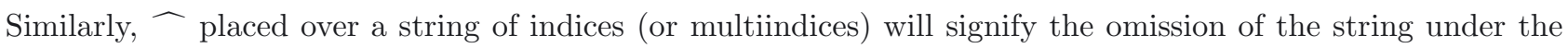
sign.

2) Let $\Omega \subset \mathbb{R}^{n}$ be a bounded open set.

- The spaces $C^{1}\left(\Omega ; \Lambda^{k}\right), W^{1, p}\left(\Omega ; \Lambda^{k}\right)$ and $W_{0}^{1, p}\left(\Omega ; \Lambda^{k}\right), 1 \leq p \leq \infty$ are defined in the usual way.

- For any $\omega \in W^{1, p}\left(\Omega ; \Lambda^{k}\right)$, the exterior derivative $\mathrm{d} \omega$ belongs to $L^{p}\left(\Omega ; \Lambda^{k+1}\right)$ and is defined by, for all $1 \leq i_{1}<\cdots<i_{k+1} \leq n$,

$$
(\mathrm{d} \omega)_{i_{1} \cdots i_{k+1}}=\sum_{j=1}^{k+1}(-1)^{j+1} \frac{\partial \omega_{i_{1} \cdots i_{j-1} i_{j+1} \cdots i_{k+1}}}{\partial x_{i_{j}}} .
$$

3) Notation for indices: The following system of notations will be employed throughout.

(i) Single indices will be written as lower case english letters, multiindices will be written as upper case english letters.

(ii) Multiindices will always be indexed by superscripts. The use of a subscript while writing a multiindex is reserved for a special purpose. See (vi) below.

(iii) $\left\{i_{1} \ldots i_{r}\right\}$ will represent the string of indices $i_{1} \ldots i_{r}$. In the same way, $\left\{I^{1} \ldots I^{r}\right\}$ will represent the string of multiindices obtained by writing out the multiindices in the indicated order.

(iv) $\left(i_{1} \ldots i_{r}\right)$ will stand for the permutation of the $r$ indices that arranges the string $\left\{i_{1} \ldots i_{r}\right\}$ of distinct indices in strictly increasing order.

(v) $\left[i_{1} \ldots i_{r}\right]$ will stand for the increasingly ordered string of indices consisting of the distinct indices $i_{1}, \ldots, i_{r}$. However, $\left[I^{1}, \ldots, I^{r}\right]$ will represent the corresponding string of distinct multiindices $I^{1}, \ldots, I^{r}$, arranged in the increasing alphabetical order, whereas $\left[I^{1} \ldots I^{r}\right]$ will represent the string of indices obtained by arranging all the distinct single indices contained in the multiindices $I^{1}, \ldots, I^{r}$ in increasing order.

(vi) For $I \in \mathcal{T}^{k}$ and $j \in I, I_{j}$ stands for the multiindex obtained by removing $j$ from $I$.

(vii) The symbol $(J ; I)$, where $J=\left\{j_{1} \ldots j_{s}\right\}$ is a string of $s$ single indices and $I=\left\{I^{1} \ldots I^{s}\right\}$ is a string of $s$ multiindices, $I^{1}, \ldots, I^{s} \in \mathcal{T}^{(k-1) s}$, will be reserved to denote the interlaced string $\left\{j_{1} I^{1} \ldots j_{s} I^{s}\right\}$. 
(viii) The abovementioned system of notations will be in force even when representing indices as subscripts of superscripts of different objects.

4) Flip: We shall be employing some particular permutations often.

Definition 7.1 (1-flip). Let $s \geq 1$, let $J \in \mathcal{T}^{s}, I \in \mathcal{T}^{l}$ be written as, $J=\left\{j_{1} \ldots j_{s}\right\}, I=\left\{i_{1} \ldots i_{l}\right\}$ with $J \cap I=\emptyset$. Let $\tilde{J} \in \mathcal{T}^{s}, \tilde{I} \in \mathcal{T}^{l}$. We say that $(\tilde{J}, \tilde{I})$ is obtained from $(J, I)$ by a 1 -flip interchanging $j_{p}$ with $i_{m}$, for some $1 \leq p \leq s, 1 \leq m \leq l$, if

$$
\tilde{J}=\left[j_{1} \ldots j_{p-1} i_{m} i_{p+1} \ldots j_{s}\right] \text { and } \tilde{I}=\left[i_{1} \ldots i_{m-1} j_{p} i_{m+1} \ldots i_{l}\right] .
$$

Definition 7.2 ( $k$-flip). Let $s \geq 1, k \geq 2$. Let $J \in \mathcal{T}^{s}, J=\left\{j_{1} \ldots j_{s}\right\}, I=\left\{I^{1} \ldots I^{s}\right\}=\left[I^{1}, \ldots, I^{s}\right]$, where $I^{1}, \ldots, I^{s} \in \mathcal{T}^{k}, I^{r}=\left\{i_{1}^{r}, \ldots, i_{k}^{r}\right\}$ for all $1 \leq r \leq s$ and $J \cap I=\emptyset$. We say that $(\tilde{J}, \tilde{I})$ is obtained from $(J, I)$ by a $k$-flip if there exist integers $1 \leq m, p \leq s$ and $1 \leq q \leq k$ such that,

$$
\tilde{J}=\left[j_{1} \ldots j_{p-1} i_{q}^{m} j_{p+1} \ldots j_{s}\right],
$$

and

$$
\tilde{I}=\left[I^{1}, \ldots I^{m-1},\left[i_{1}^{r} \ldots i_{q-1}^{r} j_{p} i_{q+1}^{r} \ldots i_{k}^{r}\right], I^{m+1}, \ldots, I^{s}\right] .
$$

Note that a $k$-flip can be seen as a permutation in an obvious way.

5) Notation for sum: For $I \in \mathcal{T}^{k s}$, where $1 \leq k \leq n$ and $1 \leq s \leq\left[\frac{n}{k}\right]$, the shorthand $\sum_{s}^{I}$ stands for the sum,

$$
\begin{gathered}
\sum_{J, \tilde{I}} \\
\begin{array}{c}
J=\left\{j_{1} \ldots j_{s}\right\}=\left[j_{1} \ldots j_{s}\right], \\
\tilde{I}=\left\{I^{1} \ldots I^{s}\right\}=\left[I^{1}, \ldots, I^{s}\right] \\
J \cup \tilde{I}=I
\end{array}
\end{gathered}
$$

Acknowledgements. We have benefitted of interesting discussions with Professor Bernard Dacorogna. Part of this work was completed during visits of S. Bandyopadhyay to EPFL, whose hospitality and support is gratefully acknowledged. The research of S. Bandyopadhyay was partially supported by a SERB research project titled "Pullback Equation for Differential Forms".

\section{REFERENCES}

[1] S. Bandyopadhyay, B. Dacorogna and S. Sil, Calculus of variations with differential forms, J. Eur. Math. Soc. 17 (2015) 1009-1039.

[2] S. Bandyopadhyay and S. Sil, Characterization of functions affine in the direction of one-divisible forms. In preparation.

[3] B. Dacorogna, Direct methods in the calculus of variations. In vol. 78 of Appl. Math. Sci. 2nd edition. Springer, New York (2008).

[4] S. Sil, Ph.D. thesis. 